\title{
NONLINEAR QUENCHING OF THE RADIOACTIVITY OF AQUEOUS SOLUTIONS OF SALTS OF NUCLIDES BY LASER ABLATION OF NANOPARTICLES OF METALS
}

\section{Stepan N. Andreev, George A. Shafeev}

A.M. Prokhorov General Physics Institute, Russian Academy of Sciences, http://www.gpi.ras.ru

Moscow 119991, Russian Federation

nauka@gpi.ru,shafeev@kapella.gpi.ru

Abstract. Laser ablation of metal nanoparticles in a solution containing radioactive nuclides, initiate their transformation into a stable state. This paper presents a brief review of studies of the dissolution of salts of radioactive uranium and cesium, where a significant acceleration of their decay is experimentally observed. Also it is proposed a possible mechanism of suppression of radioactivity, for example, salts of cesium. The obtained results allow us to hope for the establishment of effective technologies for disposal of liquid radioactive wastes containing cesium-137 and other dangerous isotopes.

Keywords: copper vapor laser, gold nanoparticles, laser ablation, radionuclides

PACS 42.62.-b; 61.46.-w; 78.66.-w

Bibliography -13 references

Received 10.05.2017

RENSIT, 2017, 9(1):65-73

DOI: $10.17725 /$ rensit.2017.09.065

\section{Contents}

1. INTRODUCTION (65)

2. LASER ABLATION OF GOLD NANOPARTICLES IN SOLUTIONS OF SALTS URANIUM AND CESIUM (66)

3. Possible mechanisms of Reduction of CESIUM RADIOACTIVITY (69)

4. Conclusion (72)

REFERENCES (72)

\section{INTRODUCTION}

Development of any area science passes certain stages. At the beginning stage of science direction are discussed the most bold theories and hypotheses and everything that could, is be checked experimentally. When science is in the process of blossoming, side hypotheses are discarded and the emphasis is on the general direction. Finally, when this direction reaches its peak and when it seems that just about everything openly and understood, appear a strange experimental results that do not fit into the framework of conventional theory: paradoxes, the resolution of which is only possible outside of that framework. Then occur return to the side directions, that were once discarded and forgotten, and sometimes breakthrough into an entirely "new dimension" of science.

So it was with mechanics, thermodynamics, optics. A similar story will be repeated still not once with different areas of science. Is no exception, and the nuclear physics.

In the period formation of nuclear physics was put forward and discussed a most different hypotheses about what is the atomic nucleus, what are its properties, is it possible to extract from it the energy. If very to simplify, we can say that in the initial phase stood out and competed two directions of development of nuclear physics. One is the high-energetic, and the other is low-energetic.

The high-energy direction promised very large dividends - are weapons and energy, and medicine. But at the same time, it carried and the danger, in the first place associated with radiation. The second direction is a low-energy - at first actively was developed, but then came to naught and was even banned. 
However, it has always attracted talented and outstanding researchers. The great Igor V. Kurchatov in 1956 he was invited to Britain, where he gave a lecture at the nuclear center in Harwell. The lecture was entitled "On the possibility of creating thermonuclear reactions in gas discharge" [1]. It was about the under his leadership experiments, on powerful electrical discharges in tubes filled with gaseous hydrogen, deuterium or helium. The strength of the discharge current has reached two million amperes. The discharge was nourish with a voltage of several dozen kilovolts.

This is a very and very small voltage by the standards of nuclear physics. Nevertheless, was reliably recorded acts of thermonuclear reactions in this discharge. Of course, now we can say that it can't be, because it can't be never, but at that time, in the far 1956, Igor Vasilievich did not hesitate to tell about it in England.

Another example is associated with the name of the famous academician Yevgeny I. Zababakhin, the creator of the russian thermonuclear weapon. The Russian Federal Nuclear Center in Snezhinsk is named after his. Yevgeny Ivanovich worked on the development of the theory of unlimited cumulation in the cavitation bubbles and mathematically strictly solved this problem [2]. His decision was not lost relevance to this day. He proved that for the energy concentration in the bubbles of a certain size there are no restrictions: neither viscosity nor thermal conductivity can lead to limiting the temperature rise in the bubble. From here directly follows the possibility of bubble fusion. Academician Zababakhin considered the idea of a simple experiment that was to demonstrate this phenomenon: in a cauldron filled with heavy water $\left(\mathrm{D}_{2} \mathrm{O}\right)$ and placed on the fire; the water boils, it bubbles; the bubbles collapse, causing a thermonuclear reaction in deuterium. Unfortunately, we have no information about the fact, whether were conducted and to what led these experiments.

There are many other examples of studies of low-energy nuclear reactions, conducted eminent scientists, including academicians of the an USSR and the RAS. Manifestations of low-energy nuclear reactions was discovered in very various experimental productions. This are and the reactions in the crystal lattice of metals induced by electrolysis, and reactions in the crystal lattice, induced by impact, the different reactions at diffusion through the membrane. This is a whole class of experiments using electric discharge, electric explosion, of ultrasound in a gas or liquid. This are reactions induced corpuscular and wave action on condensed media, and finally, reactions in biological systems.

Summarizing the known experimental data we can conclude that low energy nuclear reactions are not a synthesis, and are not a decay, but apparently, this are some collective nuclear transformations, which take place at energies unacceptably low for conventional nuclear reactions, and give the change of the isotopic composition, as well as quite a large heat generation in the full absence of residual radioactivity.

\section{LASER ABLATION OF GOLD NANOPARTICLES IN SOLUTIONS OF SALTS URANIUM AND CESIUM}

At the Prokhorov Institute of General Physics of RAS, in the laboratory of macrokinetics of non-equilibrium processes of the Scientific center of wave researches (a branch GPI) in the recent years intensively investigate ablation (removal of the substance from the surface by a laser pulse) of solids in the liquid. The basis of this phenomenon is light-hydraulic effect, discovered by G.A. Askar'yan, A.M. Prokhorov, 


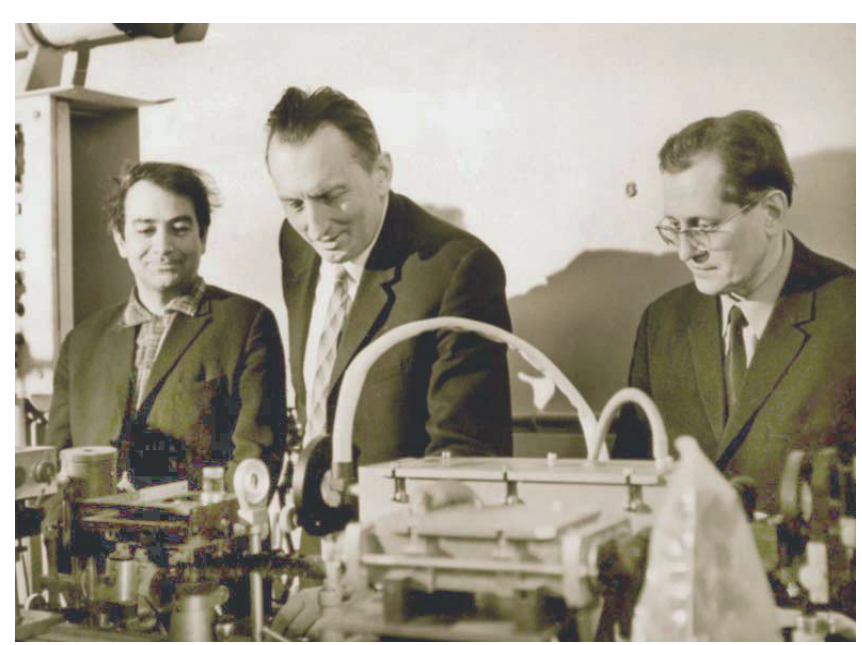

Fig. 1. G.A. Askar'yan, A.M. Prokhorov and G.P. Shipulo (left to right) beside experimental setup for the study light-bydraulic effect.

and G.P. Shipulo (Fig. 1) at the beginning of the laser era in $1963[3,4]$.

If the laser beam acts on the absorptive target, located in a transparent fluid, at a sufficiently high intensity of laser exposure, on the target surface the nanorelief arises, and into the surrounding liquid stand out nanoparticles from the material of the target (Fig. 2). In principle, this technique allows to generate nanoparticles of any kind - metallic, dielectric, semiconductor.

It was shown that laser irradiation of nanoparticles of metals in aqueous solutions of salts of uranium leads to a significant

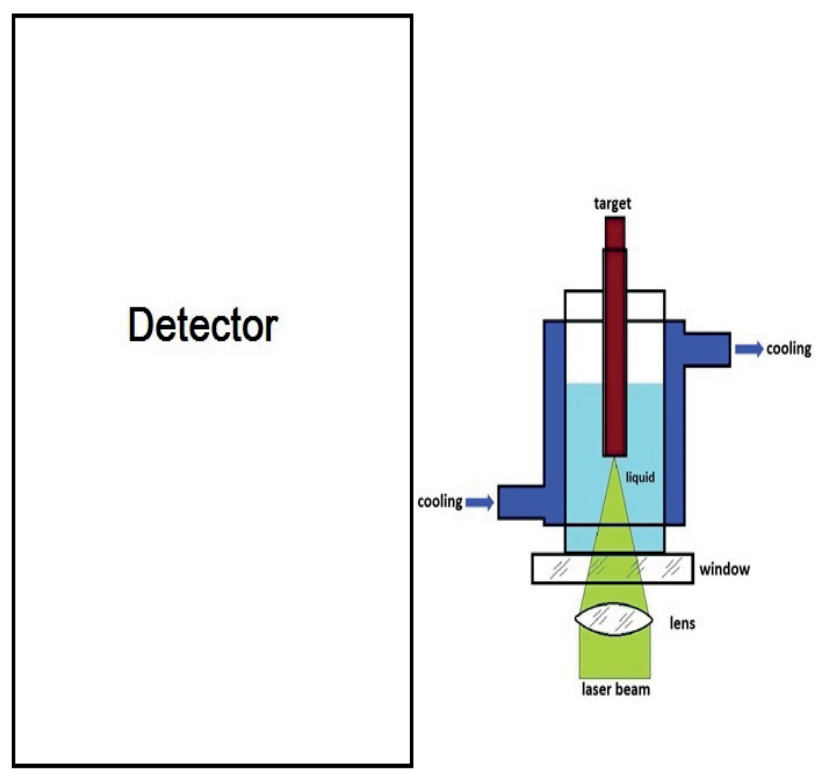

Fig. 2. Laser ablation of solids in liquid. deviation of activity of the radionuclides of this family from the equilibrium values $[5,6]$. Change of activity of radionuclides occurs in the process of laser irradiation of metal targets in aqueous solutions of salts of uranium. Herewith an acceleration of the spontaneous $\alpha$ - and $\beta$-decay nuclides belonging to the family of uranium-238 is observed. This effect was investigated in a wide range of wavelengths of the laser radiation and pulse durations [7, 8]. Laser ablation of targets in aqueous solutions of salts of nuclides accompanied by the formation in solution of nanoparticles of the target material and, apparently, is a necessary condition for changes in the activity of the nuclides. This conclusion can be drawn from observing the growth of activity of a number of nuclides (thorium-234) for a long time after laser irradiation - up to 100 days [9].

In the present work we will focus on the irradiation by the pulsed laser of an aqueous solution of a salt of cesium-137 (Fig. 3) with placed in it by gold nanoparticles (Fig. 4) which form in water a fairly stable colloidal solution [10].

Glass cuvette with an aqueous salt solution of the radioactive isotope cesium-137, in which added gold nanoparticles at a concentration of $10^{12}$ units per milliliter, was irradiated through

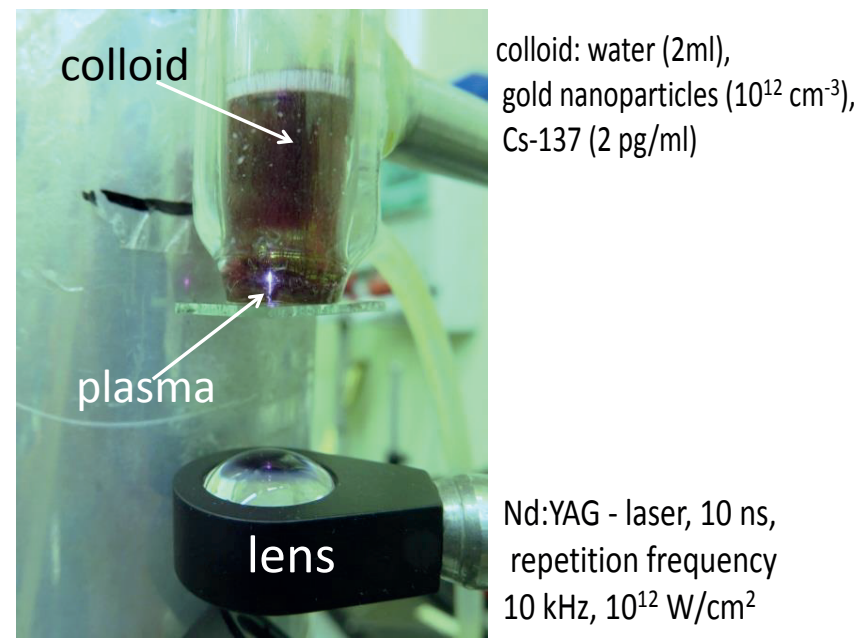

Fig. 3. The irradiation of pulsed laser radiation of an aqueous solution of a salt of caesium-137 placed in him by the gold nanoparticles. 


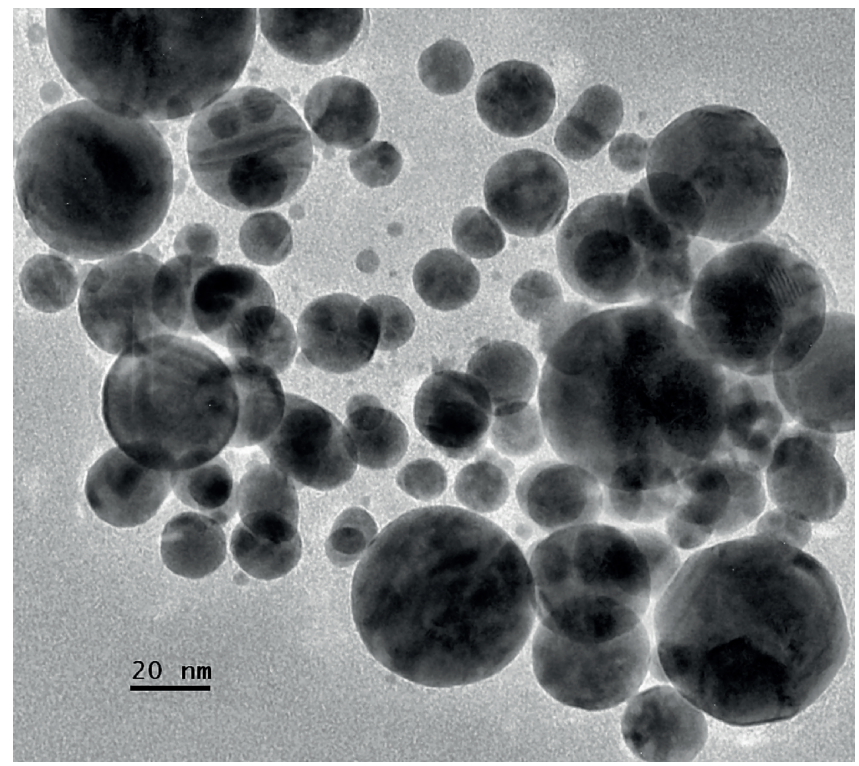

Fig. 4. Gold nanoparticles obtained by ablation of a gold target in aqueous solution of salt of cesium-137.

the transparent bottom by the pulses of the copper vapor laser. The energy of each pulse was $2 \mathrm{~mJ}$, duration $10 \mathrm{~ns}$, repetition frequency $10 \mathrm{kHz}$. The characteristic time of irradiation - ten hours. In order to the transparent bottom of the cuvette is not quickly destroyed, the laser beam is focused not at the same place, and scanned over the surface of the cuvette. External cooling of the cuvette with running water prevented the boiling in it a radioactive solution.

In contrast to the nuclides of family of uranium-238, at cesium-137 there is only one

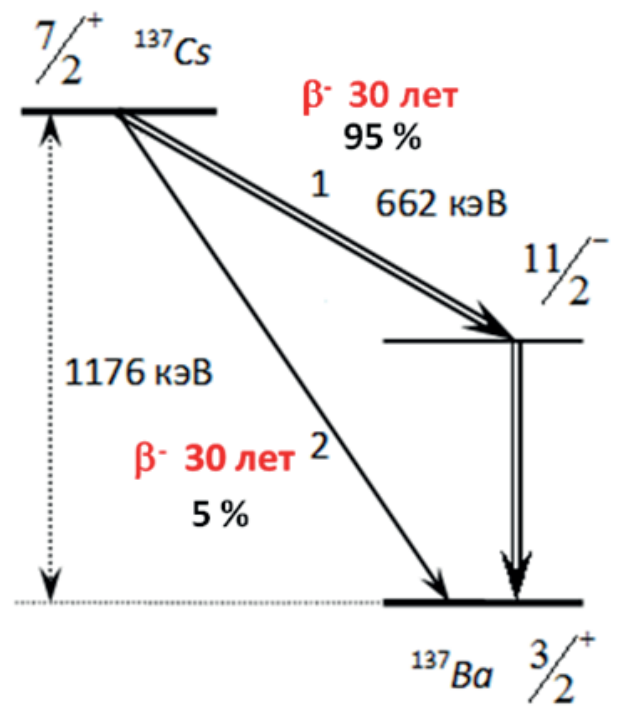

Fig. 5. Diagram of the beta decay of the isotope cesium-137. channel of decay - $\beta$-decay. There are two channels of $\beta$-decay (Fig. 5). The first channel, which is realized with a probability of $95 \%$, is the beta decay of cesium-137 to an excited state of barium-137, the loss of excitation by a nucleus of barium results in the emission of gamma-quant with energy $662 \mathrm{keV}$. I.e., in fact, in the experiment measured the number of excited nuclei of barium. But because this transition is very rapid compared to beta decay (half-life of cesium-137 is 30 years), in fact, the intensity of the gamma-quants determines the concentration of nuclei of caesium-137. Measurement of the intensity of the flux of gamma-quants is a very reliable method of measuring the concentration of nuclei of caesium, as gamma-quanta with energy $662 \mathrm{keV}$ are not absorbed into either the liquid or the nanoparticles or glass of cuvettes.

There is a second channel of the beta decay of cesium-137 that is implemented with a probability of $5 \%$. In it beta-electron is emitted from the nucleus of a cesium with energy of order $1 \mathrm{MeV}$, and the nucleus of the barium is in the unexcited, ground state. This channel is nonradiative and cannot be used for measurements in this scheme of the experiment for the reason that electrons with an energy of about $1 \mathrm{MeV}$ can not leave the cuvette - they are completely braked in water, not reaching the walls of the cuvette.

Gamma radiation from the fixed cuvette was measured by semiconductor gamma spectrometer Ortec-65195-P with an accuracy of $\pm 5 \%$ continuously for 15 hours before laser irradiation, 10 hours during the experiment, and 15 hours after irradiation.

A typical gamma spectrum from the cuvette with a solution of caesium-137 is shown in Fig. 6. The solution activity was measured by area under the spectral peak of gamma-quant with energy $662 \mathrm{keV}$ using a special program. Activity values were saved in computer memory 


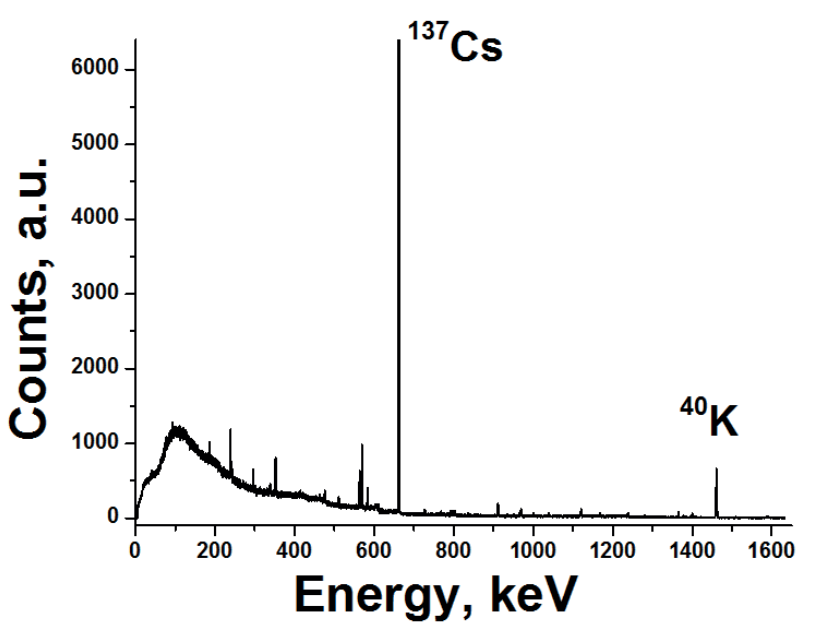

Fig. 6. A typical gamma spectrum from the cuvette with a solution of cesium-137.

with a frequency of 1 time per second, and the time of signal accumulation was selected such that the measurement error did not exceed $0.5 \%$. The peak contrast relative to the background was 120:1.

Preliminary measurements of gamma spectrum of the cuvette, with radioactive cesium-137 for 15 hours before laser irradiation showed that the concentration of cesium in the cuvette during this time has not changed (which, on the one hand, to be expected, because the period of half-decay of cesium-137 is a lot more observation time, and on the other hand, this fact indicates the absence of possible care paths of caesium from the cuvette).

The main result of the experiment, conducted jointly with specialists from Dubna, lies in the detection by they the monotonic decrease of the flux of gamma quanta with energy $662 \mathrm{keV}$ on $5 \%$ at the time of laser irradiation of the cuvette, with a solution of radioactive cesium-137 for 10 hours (Fig. 7).

Subsequent measurements of gamma spectra for 15 hours showed that after switching off the laser the gamma activity of the cuvette with the solution remains at a constant level.

Thus, for 10 hours of laser exposure, the concentration of radioactive cesium in the cuvette decreased by $5 \%$. Formally, this corresponds to the acceleration beta-decay

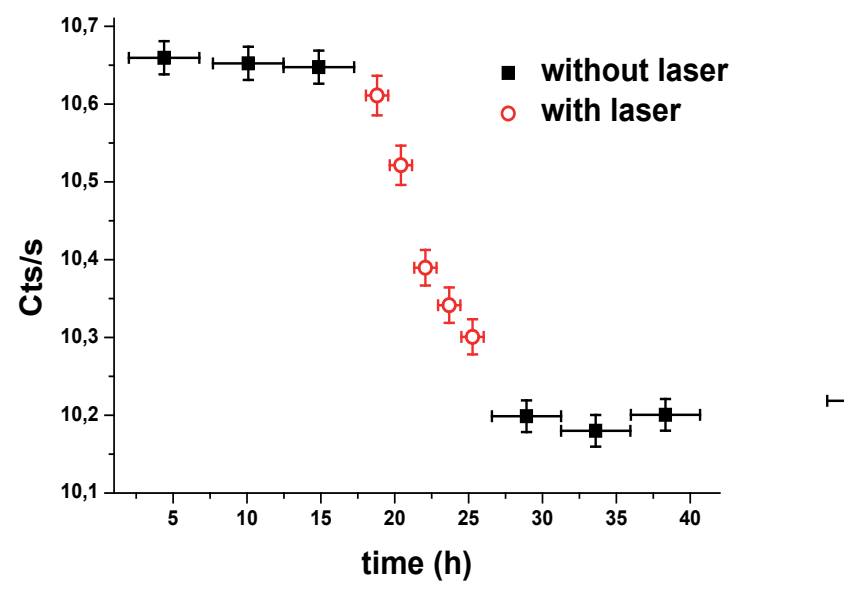

Fig. 7. The dependence of the intensity of the flux of gamma rays with energy of $662 \mathrm{Kev}$ from the time before, during, and after irradiation by laser pulses of the cuvette with a solution of radioactive cesium- 137 .

of cesium-137 is not less, than 1200 times. However, in reality, here we are faced with manifestation of the another nuclear process. The effect we called "nonlinear quenching of the radioactivity of cesium-137" - similar to the quenching of luminescence in laser crystals.

\section{POSSIBLE MECHANISMS OF REDUCTION OF CESIUM RADIOACTIVITY}

The discovered effect is not the acceleration of beta-decay. This is easily seen by following the method of the proof "by contradiction". Without going into the mechanism of the process, assume that during laser exposure, for whatever reason, the acceleration of betadecay of the nucleus of caesium-137 occur. Herewith this realizes the decay channel into the excited state of the barium nucleus. Now its half-decay period is already not 30 years, and just 9 days (this figure is obtained taking into account the fact that for 10 hours, the decrease of the radioactivity of the solution was $5 \%$ ). What then will be the result? The nuclei of cesium will go to an excited level of the nuclei of barium 1200 times faster. Consequently, the intensity of the flux of gamma quanta with energy $662 \mathrm{keV}$ should increase by 1200 times. However, gamma-ray spectrometer does not register that. On the contrary, it shows a 
monotonous decrease in the intensity during laser irradiation. So, talk about the acceleration of the beta decay of cesium in this case is incorrect.

Consider another possibility, when beta decay accelerated by non-radiative channel of the caesium into the ground state of barium. To explain the experimental data, its half-life must equal total of 11 hours, as the radiationless transition occurs only in $5 \%$ of cases. Beta decay of cesium on this channel will not give an increase in the intensity of gamma quanta with energy $662 \mathrm{keV}$, instead there will be a large number of electrons with energy around $1 \mathrm{MeV}$. These electrons, scattered on the surrounding atoms will give to gamma-quanta of bremsstrahlung radiation with a continuous spectrum of energies from a high of $1176 \mathrm{keV}$ and below. Their emergence one may register with the help of gammaspectrometer, however, this task is connected with considerable experimental difficulties. In particular, it is necessary to eliminate the effect of cosmic gamma background on the measurement results. Work in this direction is underway, but so far the achieved measurement accuracy does not allow to make certain conclusions.

The third possibility of reducing the radioactivityof cesiumliesintheimplementation of nuclear reactions during laser irradiation of solution. As we know from nuclear physics, nuclear reactions must overcome the Coulomb barrier of repulsion of the positively charged nuclei involved in the reaction. This requires a temperature of millions of degrees, or a highenergy accelerator. It is obvious that in our experiment none of these possibilities is not possible. Therefore, about the occurrence of the usual nuclear reactions in this case is not out of the question.

However, we cannot exclude the possibility of exotic nuclear processes, the theory of which develop in the works of doctors of Phys\&Math Sciences V.I. Man'ko [11] and V.I. Vysotskii [see, for example, on pages 21-36 of this issue and the bibliography in it]. From their theory it follows that for the implementation of exotic low-energy nuclear reaction it is necessary that the nuclei were correlated in a coherent quantum state. Recall that these are the states in which fluctuations of momentum and coordinates of a quantum particle are not independent, and therefore, the ratio of uncertainty of Heisenberg is written for them in the form of an equation of the SchrödingerRobertson [12, 13]. It takes into account the correlation coefficient, which in the usual uncorrelated quantum states is equal to zero (then this formula reduces to the ratio of the Heisenberg uncertainty), but may be and very close to unity. Then the product of the fluctuations of the coordinate and momentum of a particle can increase substantially. Formally, this corresponds to increasing the "effective" Planck's constant by several orders of magnitude, which in turn leads to a multiple increase in the probability of tunneling of a quantum particle through the Coulomb barrier.

There is a fundamental difference of correlated particles in the coherent condition compared with the particles having high kinetic energy.

If the particle with high kinetic energy collides with an atomic nucleus, this nucleus, as a rule, enters an excited state, the decay of which is usually accompanied by the emission of gamma quanta, neutron yield, electrons, or other nuclear particles. As a result, the kernel acquires the residual radioactivity.

If the particle is in a coherent correlated state, then she may have high energy because of the fluctuations on very short time, that is determined by the uncertainty relation Schrödinger-Robertson. A particle with such energy would react with the nucleus, but 
this happens only if the excited state of the nucleus, which arises herewith, relaxed during the time less than the lifetime of fluctuations of the energy of the particle.

I.e. not every nuclear reaction can be come true, but only one in which the products have a very short relaxation time to the ground state. In other words, those new nuclei that occur in these exotic nuclear processes do not have residual radioactivity, i.e., are stable.

As shown by V.I. Vysotskii, for the formation of coherent correlated state the quantum particle must be in a potential pit, the walls of which experience a rapid changes. Potential pit must either monotonically expand or shrink, or experience periodic perturbations. An example of such a potential pit can serve as a nano-cavity in the crystal lattice of a solid body subjected to shock compression or rupture. Another example, of direct relevance to experiment [10], is a nano-sized cavitation bubble in the liquid, the volume of which fluctuates under the action of external forces. Such nanoscale bubbles appear in laser irradiation of colloidal solution of gold nanoparticles.

It is possible to give some estimates. The average size of the nanoparticles in the experiment [10] is of the order of $10 \mathrm{~nm}$. With long-term laser exposure the nanoparticles are fragmented and their size can be smoothly reduced to $3 \mathrm{~nm}$. The concentration of nanoparticles in solution $-10^{12}$ units per $\mathrm{ml}$, the average distance between them - of about 1 micron.

What happens to a nanoparticle trapped in the focus of the laser pulse? It absorbs the laser energy, heats up and becomes the center of nucleation - on it arise nanoscale cavitation bubble. The surface of these bubbles are charged. With the growth of nanobubble on its surface there is a separation of charges, and inside it formed a sufficiently large electric field, which causes electrical breakdown of a gas-vapor mixture inside the bubble and the formation of a plasma consisting primarily of electrons and protons. The resulting plasma then absorbs the laser radiation, which leads to further growth of the bubble. According to our calculations, the electric field inside the bubble can reach values of the order of $10 \mathrm{kVcm}^{2}$. This is sufficient to breakdown the gas.

We conducted special experiments in which we measured the duration of the individual flashe from nanobubble and the spectrum of its radiation. It is obtained that the flash duration is around $10 \mathrm{~ns}$ - this coincides with the duration of a single laser pulse. Spectrum of flash continuous, that matches the spectrum of the bremsstrahlung of the electrons in the plasma. Evaluation of plasma temperature give a value of the order of several electron volts.

It should be emphasized that the characteristic distance between nanobubbles of the order of $1 \mu \mathrm{m}$ is large enough to consider them independent from each other. It is well known that the cavitation processes are most intense when the bubbles are separated from each other. Once they merge, the efficiency of cavitation drops sharply. From this follows an important condition for the intensity of the laser pulses: it must be high enough for the formation of cavitation bubbles on the gold nanoparticles, but not to exceed such values, when the bubbles merge with each other.

So, as a result of the impact of laser pulse on gold nanoparticle is formed nanobubble containing electron-proton plasma. In fact, the bubble is proton potential well, the walls of which are deformed due to the fact that the bubble grows or shrinks. Therefore, the protons can be in a coherent correlated States. Fluctuations of the proton energy in these States can be sufficient to proton react with a nearby nucleus of caesium-137 with the 
formation of non-radioactive barium-138 in ground state.

Thus, a third possible mechanism for reducing the radioactivity of a solution of cesium lies in the occurrence of nuclear reactions between protons, which are in coherent correlated states in nanobubbles, and nuclei of caesium-137, with the formation of non-radioactive barium-138.

\section{CONCLUSION}

Obtained results extend the list of radionuclides $\left.{ }^{232} \mathrm{Th},{ }^{238} \mathrm{U},{ }^{3} \mathrm{H}\right)$, the accelerated decay of which can be initiated with the aid of laser radiation. These data are of practical interest, since cesium-137 is one of the most hazardous radionuclides due to its rapid spread in water and soil.

At the present time there are approximately two hundred nuclear power plants, in which operate almost 450 power unit. Many of them have almost exhausted their resource and prepare for decommissioning. In this regard, the acute question of disposal of liquid radioactive waste (RW), first of all, water under the reactor. Currently, the solution to the problem of disposing of radioactive waste in fact confines to their long-term storage, in which radioactive isotopes disintegrate naturally over tens or hundreds of years. In fact — is shifting the solution of environmental problems of the present generation on the shoulders of its immediate and distant descendants. Even more acute problem of disposal of radioactive waste arises in case of emergencies at nuclear facilities (Chernobyl, Fukushima), and in the case of military conflicts with the use of nuclear weapons, as a result of which extensive territories undergo by radiation contamination. Therefore, the development of technologies accelerated deactivation of the radiation contaminations and the utilization of radioactive waste is an urgent and priority task of nuclear physics.
The relative simplicity of the technological realization of the laser quenching of radioactivity gives a real possibility of its use for the decontamination of radiological contaminants and adjustment of the composition of radioactive waste of atomic energetics.

\section{REFERENCES}

1. Kurchatov IV. About possibility of creation of thermonuclear reactions in gas discharge. Advances in physical Sciences, 1956, 59(4):603-618.

2. Zababakhin EI, Zababakhin IE. Yavleniya neogranichennoy kumulyatsii [The phenomena of unlimited cumulation]. Moscow, Nauka Publ., 1988, p. 161.

3. Askar'ian GA, Prokhorov AM, Chipulo GP, Chanturia GF. Luch OKG v zhidkosti [The ray of optical quantum generator in fluid]. Pis'ma v Zhetf, 1963, 44(6):2180-2182 (in Russ.).

4. Askar'ian GA, Prokhorov AM, Chipulo GP. Sbetogidravlicheskiy effekt [The light-hydraulic effect]. Diploma No. 65. Priority 28.2.1963. Otkrytiya, izobreteniya, promyshlennye obraztsy, tovarnye znaki [Discoveries, inventions, industrial designs, trademarks], 1969, 19:4 (in Russ.).

5. Simakin AV, Shafeev GA. Initiation of nuclear reactions under laser irradiation of Au nanoparticles in the aqueous solution of Uranium salt. Appl. Phys. A, 2010, 101(1):199.

6. Simakin AV, Shafeev GA. The influence of laser irradiation of nanoparticles in aqueous solutions of uranium salts on the activity of the nuclides. Quantum electronics, 2011, 41(7):614.

7. Barmina S, Sukhov IA, Lepekhin NM, Priseko YuS, Filippov VG, Simakin AV, Shafeev GA. The use of copper vapor lasers for controlling activity of uranium isotopes. Quantum electronics, 2013, 43(6):591-596. 
8. Shafeev GA, in: Uranium: Characteristics, Occurrence and Human Exposure. Ed. by A. Ya. Vasiliev, M. Sidorov. New York, Novapubl. Inc., 2012, pp 117-153.

9. Kirichenko NA, Simakin AV, Shafeev GA. Long-term variation in nuclide activities under laser ablation of metallic targets in aqueous solutions of uranium salt. Phys. Wave Phenomena, 2014, 22(2):81.

10. Barmina EV, Simakin AV, Shafeev GA. Laser-induced decay of cesium-137. Quantum electronics, 2014, 44(8):791-792.

11. Dodonov VV, Manko VI. Invarianty i evolutsiya nestatsionarnylh kvantovykh sistem [Invariants and evolution of nonstationary quantum systems]. Trudy FLAN. Moscow, Nauka Publ., 1987, 183:286-351.

12. Robertson HP. A general formulation of the uncertainty principle and its classical interpretation. Physical Review, 1930, 35(5):667.

13. Schrödinger E. Zum Heisenbergschen Unscharfeprinzip. Berl. Königlich Akad. Wiss., 1930, 24:296-303. 